\title{
POLYNOMIAL RICCATI EQUATIONS WITH ALGEBRAIC SOLUTIONS
}

\author{
HENRYK ŻOŁĄDEK \\ Institute of Mathematics, University of Warsaw \\ Banacha 2, 02-097 Warszawa, Poland \\ E-mail: zoladek@mimuw.edu.pl
}

\begin{abstract}
We consider the equations of the form $\frac{d y}{d x}=y^{2}-P(x)$ where $P$ are polynomials. We characterize the possible algebraic solutions and the class of equations having such solutions. We present formulas for first integrals of rational Riccati equations with an algebraic solution. We also present a relation between the problem of algebraic solutions and the theory of random matrices.
\end{abstract}

1. Introduction. The Riccati equations in complex domain

$$
\frac{d y}{d x}=p(x) y^{2}+q(x) y+r(x)
$$

where $p, q, r$ are rational functions, are interesting from the dynamical and algebraic point of view. They describe the evolution of directions of solutions of 2 -dimensional linear differential systems

$$
\frac{d z}{d t}=A(x) z
$$

with meromorphic matrices $A(x)$, or of second order linear differential equations

$$
\frac{d^{2} w}{d x^{2}}=a(x) \frac{d w}{d x}+b(x) w
$$

with meromorphic $a(x), b(x)$.

Some Riccati equations are associated with non-autonomous periodic systems via the substitution $x=e^{i t}, t$ real (see [Zo3]).

In the book of I. Kaplansky [Ka] there is a proof that the Airy equation

$$
\frac{d^{2} w}{d x^{2}}=x w
$$

2000 Mathematics Subject Classification: Primary 12H05; Secondary 34C05, 58F21.

Key words and phrases: Riccati equation, algebraic solution, random matrix.

The work was supported by Polish KBN Grant No 2 P03A 04115.

The paper is in final form and no version of it will be published elsewhere. 
is not solvable in quadratures. The proof is eventually reduced to showing that the Riccati equation associated with (1.3)

$$
\frac{d y}{d x}=x-y^{2}
$$

does not have invariant algebraic curves of the form $y=u(x)$ or $y^{2}+u(x) y+v(x)=0$, where $u, v$ are rational functions. Similarly one can show that the Bessel equation

$$
x^{2} \frac{d^{2} w}{d x^{2}}+x \frac{d w}{d x}+\left(x^{2}-\nu^{2}\right) w=0
$$

does not define a generalized Liouville extension of the field $\mathbb{C}(x)$ for $\nu^{2} \neq \frac{1}{4}$ (in $[\mathrm{MoRu}]$ this is proved using Stokes operators).

Therefore the problem of existence of invariant algebraic curves $K(x, y)=0$ for the equation (1.1) is interesting. Each such curve represents a solution $y=\varphi(x)$ of the equation, this solution is an algebraic function. (When $p, q, r$ in (1.1) are not polynomials, then there are invariant lines $x=x_{i}$, where $x_{i}$ are poles of $(p, q, r)(x)$. However we skip such curves, we are interested in non-vertical curves.)

Using the monodromy group (or the differential Galois group associated with the equation (1.2)) one can show that the minimal degrees $\operatorname{deg}_{y} K$ can be equal to $1,2,4,6$ or 12 (J. Drach, J. A. Weil). Using this J. J. Kovacic [Ko] has constructed an algorithm for solving the linear equation (1.2) in generalized quadratures. The cases with minimal degrees 4, 6, 12 correspond to the monodromy groups isomorphic to the groups of rotations of the regular tetrahedron, cube and icosahedron respectively.

Some algebraists treat the problem of algebraic solutions to equations like (1.1) in a slightly different way. Assume that in (1.1) we have $p(x)=P(x) / S(x), q(x)=Q(x) / S(x)$ and $r(x)=R(x) / S(x)$, where $P, Q, R, S$ are polynomials. They introduce the derivation (polynomial vector field) of the ring $\mathcal{R} \stackrel{d f}{=} \mathbb{C}[x, y]$ by the formula

$$
V=S(x) \frac{\partial}{\partial x}+\left[P(x) y^{2}+Q(x) y+R(x)\right] \frac{\partial}{\partial y} .
$$

A polynomial derivation is called simple if the $\operatorname{ring} \mathcal{R}$ does not contain proper (i.e. $\neq 0, \mathcal{R}$ ) differential ideals.

Of course, the simplicity of $V$ means the non-existence of affine invariant algebraic curves for the polynomial vector field defined by the derivation.

The idea of writing this paper came to me after reading the recent paper [MaMoNo] by A. Maciejewski, J. Moulin-Ollagnier and A. Nowicki. There the authors have obtained the following results about simplicity of polynomial derivations of the form

$$
V_{P}=\frac{\partial}{\partial x}+\left[y^{2}-P(x)\right] \frac{\partial}{\partial y},
$$

where $P(x)$ is a polynomial. (Note that any derivation (1.4) with $S(x) \equiv 1$ can be reduced to the form (1.5).)

The authors of [MaMoNo] have proved that:

- any invariant algebraic curve is of the form $y=w(x)$ with rational $w$;

- if $\operatorname{deg} P$ is odd, then $V_{P}$ is simple; 
- if $P$ is quadratic, of the form $x^{2}+a$, then $V_{P}$ is non-simple if and only if $a$ is an odd integer.

(In fact, in [MaMoNo] the field of coefficients $k$ is not just $\mathbb{C}$, but an arbitrary algebraically closed field of characteristic zero. In the present paper we shall avoid this kind of abstractness.)

The aim of this work is to provide analytic proofs of the results of [MaMoNo] and to generalize them, e.g. give more precise characterization of rational solutions and of non-simple derivations. This is done in Sections 3 and 4 below.

After the first version of the paper was submitted for publication, I have learned from J. A. Weil about the paper [Vi] of R. Vidunas (student of M. van der Put). In the latter paper essentially the same results as mine in Theorems 1 and 2 (in Section 3 below) were proved. However, Vidunas's proofs are highly complicated and abstract (they use the language of categories and schemes); I was not able to pass through them. My proofs are analytic geometrical and use geometry of the configuration space. Apparently, the authors of [MaMoNo] also were not aware of Vidunas's work.

In Section 2 we present one result about possible forms of first integrals for rational Riccati equations with an algebraic solution. These formulas were given in [Zo1, Lemma $5]$ and in $\left[\mathrm{Zo}_{\mathrm{o}}\right]$ (in [ $\left.\mathrm{Zo} 2\right]$ one can also find analogous formulas for the Liénard equations). Since the result seems to be interesting and its proof in [Zo1] was only sketched and incomplete, I use the opportunity to present it here with the full proof.

There is an interesting connection of the problem presented here with the theory of random matrices. Namely the conditions for poles of rational solutions to (1.5) are the same as the conditions for the most probable system of eigenvalues of a random Hermitian matrix. In the case of quadratic $P$ the poles are zeroes of the Hermite polynomials, which are associated with the Gaussian Unitary Ensemble (see [De]). In Section 5 we use the results about asymptotic behaviour of random matrices of large dimension to prove a result (Theorem 3 below) about asymptotic distribution of poles of rational solutions to the Riccati equations.

2. First integrals. Knowing one solution $y=\varphi(x)$ to equation (1.1), i.e. $\varphi^{\prime}(x) \equiv$ $p(x) \varphi^{2}(x)+q(x) \varphi(x)+r(x)$, one can integrate the equation. One puts $y_{1}=y-\varphi(x)$ and obtains the Bernoulli equation

$$
\frac{d y_{1}}{d x}=p(x) y_{1}^{2}+q_{1}(x) y_{1}
$$

with

$$
q_{1}(x)=q(x)+2 p(x) \varphi(x) .
$$

The equation (2.1) has the first integral

$$
H=\frac{1}{y_{1}} e^{\int q_{1}}+\int e^{\int q_{1}} p .
$$

It is interesting to know what the formula (2.2) leads to, when the known solution $y=\varphi(x)$ is an algebraic function which satisfies an algebraic equation $K(x, \varphi(x)) \equiv 0$. The curve

$$
K(x, y)=0
$$


is an invariant curve for the equation (1.1), and for the vector field (1.4) as well. We denote by $\operatorname{deg}_{y} K$ the degree of the polynomial $K(x, y)$ with respect to $y$. We assume also that the curve $K=0$ does not contain the vertical lines $x=x_{j}$ as components, thus $\operatorname{deg}_{y} K>0$.

We divide the further analysis into four cases:

(a) One rational solution. This case means

$$
\max _{K} \operatorname{deg}_{y} K=1
$$

i.e. there is only one algebraic solution and it is of the form

$$
y=s(x),
$$

where $s(x)$ is a rational function.

Proposition 1. If the Riccati equation (1.1) has a rational solution (2.3), then it has a first integral of the Darboux-Schwarz-Christoffel type

$$
H=\frac{1}{y-s(x)} e^{g(x)} \prod\left(x-x_{i}\right)^{a_{i}}+\int^{x} e^{g(u)} \prod\left(u-x_{i}\right)^{a_{i}-m_{i}} P(u) d u,
$$

where $P(x)$ is a polynomial, $g(x)$ is a rational function and $x_{i}, a_{i} \in \mathbb{C}, m_{i} \in \mathbb{Z}_{+}$.

Proof. The functions $p(x)=P(x) / \prod\left(x-x_{i}\right)^{m_{i}}, \varphi(x)=s(x)$ and $q_{1}(x)$ are rational with poles in the set $\left\{x_{1}, \ldots, x_{k}\right\}$, containing the set of poles of the right-hand side of (1.1). The integral $\int q_{1}$ in (2.2) takes the form $\sum a_{i} \log \left(x-x_{i}\right)+g(x)$ with rational function $g$. Therefore the formula (2.4) follows.

(b) Two rational solutions. This case means

$$
\min _{K} \operatorname{deg}_{y} K=1, \max _{K} \operatorname{deg}_{y} K=2,
$$

i.e. there are exactly two algebraic solutions and they are rational,

$$
y=s_{1}(x), y=s_{2}(x),
$$

$s_{1,2}$ rational.

Proposition 2. If the Riccati equation (1.1) has rational solutions (2.5), then it has a first integral of the Darboux type

$$
H=\frac{y-s_{2}(x)}{y-s_{1}(x)} e^{g(x)} \prod\left(x-x_{i}\right)^{b_{i}} .
$$

Proof. Assume that besides the rational solution $y=s(x)=s_{1}(x)$, as in Proposition 1 , there is another rational solution $y=s_{2}(x)$. Without loss of generality we can assume that $s_{1}(x) \equiv 0$ and $y=y_{1}$. We have

$$
\begin{aligned}
\frac{d}{d x}\left(e^{\int q_{1}} \cdot \frac{1}{s_{2}}\right) & =\frac{d}{d x}\left(\left.e^{\int q_{1}} \cdot \frac{1}{y_{1}}\right|_{y_{1}=s_{2}(x)}\right)=e^{\int q_{1}} \cdot\left(\frac{-y_{1}^{\prime}}{y_{1}^{2}}+\frac{q_{1}}{y_{1}}\right) \\
& =e^{\int q_{1}} \cdot(-p) .
\end{aligned}
$$

Thus $H=\left(\exp \int q_{1}\right) \cdot\left(1 / y_{1}-1 / s_{2}(x)\right)$ in $(2.2)$, which leads to the formula (2.6).

(c) One hyperelliptic solution. This is the case when

$$
\min _{K} \operatorname{deg}_{y} K=\max _{K} \operatorname{deg}_{y} K=2,
$$


i.e. there is only one invariant algebraic curve and it is hyperelliptic

$$
(y+t(x))^{2}=s(x),
$$

$s(x), t(x)$ rational.

Proposition 3. If the Riccati equation (1.1) has a solution given by (2.7), then it has a first integral of the Darboux-hyperelliptic type

$$
H=\frac{y+t(x)+\sqrt{s(x)}}{y+t(x)-\sqrt{s(x)}} \exp \left[\int^{x} w(u) \sqrt{s(u)} d u\right],
$$

where $w(x)$ is rational.

Proof. After the change $y \rightarrow y+t(x)$ we can assume that $\varphi(x)=\sqrt{s(x)}$. Its substitution to the initial Riccati equation (1.1) gives $\frac{s^{\prime}}{2 \sqrt{s}}=p s+q \sqrt{s}+r$, i.e. $q=\frac{s^{\prime}}{2 s}$ and $q_{1}=\frac{s^{\prime}}{2 s}+2 p \sqrt{s}$. Therefore in $(2.2)$ we have

$$
\begin{aligned}
H & =\frac{1}{y_{1}} \cdot \sqrt{s} e^{2 \int p \sqrt{s}}+\int \sqrt{s} e^{2 \int p \sqrt{s}} \cdot p \\
& =\frac{1}{y_{1}} \cdot \sqrt{s} e^{2 \int p \sqrt{s}}+\frac{1}{2} e^{2 \int p \sqrt{s}}=\frac{1}{2} e^{2 \int p \sqrt{s}} \cdot \frac{y+\sqrt{s}}{y-\sqrt{s}} .
\end{aligned}
$$

(d) Algebraic solutions with $\geq 3$ branches. Here we have

$$
\max _{K} \operatorname{deg}_{y} K \geq 3 .
$$

Note that the invariant algebraic curve $K=0$ can have several irreducible components.

Proposition 4. Assume that the Riccati equation (1.1) has an invariant algebraic curve satisfying (2.9). Then all its solutions are algebraic and there is a first integral in the form of rational function in $x, y$.

Proof. In the case $\operatorname{deg}_{y} K>2$ we firstly show that the monodromy group Mon of the Riccati equation is finite. Here Mon consists of the Möbius transformation of a Riemann sphere $\widehat{\mathbb{C}} \simeq \mathbb{C} P^{1} \times\left\{x_{0}\right\}$ defined by means of prolongations of solutions (which start at a distinguished fiber $\mathbb{C} P^{1} \times\left\{x_{0}\right\}$ ) along loops in the $x$-plane which avoid poles of the equation.

The monodromy group is finite, because it has invariant set $A$ of cardinality $\geq 3$ (the points of intersection of the invariant curve with the fiber $x=x_{0}$ ) and the only Möbius transformation with fixed three points is the identity. Indeed, in the exact sequence $e \rightarrow G \rightarrow$ Mon $\rightarrow S(A)$ (where $S(A)$ is the permutation group of the set $A$ ) the group $G$ is trivial.

Therefore the solutions have finite monodromy. In such case it is enough to show that they have regular singularities, of power growth (i.e. not like $e^{-1 / x^{2}}$ ). Then all solutions turn out to be algebraic. (It is well known that, if a polynomial vector field has infinitely many algebraic invariant curves, then it has a rational first integral.)

The singularities of solutions lie at the points $x=x_{j}$. The lines $\left\{x=x_{j}\right\} \subset \mathbb{C}^{1} \times \mathbb{C}^{1}$ are invariant lines of the polynomial vector field $V=S(x) \frac{\partial}{\partial x}+\left(P(x) y^{2}+Q(x) y+R(x)\right) \frac{\partial}{\partial y}$ associated with the Riccati equation. This field defines a holomorphic foliation $\mathcal{F}$ (by complex phase curves) of $\mathbb{C} P^{1} \times \mathbb{C} P^{1}$. The singular points of the foliation $\mathcal{F}$ lie in the 
projective lines $\left\{x=x_{j}\right\} \times \mathbb{C} P^{1}$ and $\{x=\infty\} \times \mathbb{C} P^{1}$. Each such line contains at most 2 singular points. Thus we shall look at the behaviour of phase curves near such singular points.

Consider one such invariant line, which we may assume to be $x=0$. The curve $K=0$ intersects it at $\geq 3$ points, counting multiplicity. Each local branch of $K=0$ represents an algebraic solution $y=\varphi(x)$, where the function $\varphi$ admits a Puiseux expansion. Firstly we perform the pull-back $x=u^{q}$, in order to remove multi-validity of the local branches of $K=0$. We get $y=c u^{l}+\ldots, l$ integer, for any such branch and the (projective) line $u=0$ contains a singular point of the foliation (induced from $\mathcal{F}$ ) through which at least 2 such branches pass. We choose a pair $y=\psi_{1}(u), y=\psi_{2}(u)$ with the greatest order of tangency. Next, we separate the branches $y=\psi_{1,2}(u)$ using a series of changes of the form $y=z u^{m}$ and/or $y=\psi(u)+z$ (where $y=\psi(u)$ is some branch). (Note that the rational Riccati form of the differential equation is preserved during such operations.) We arrive at the situation with the branches $z=\chi_{1,2}(u)=c_{1,2}+O(u)$ and $c_{1} \neq c_{2}$. The third branch $z=\chi_{3}(u)$ of $K=0$ will have the form: either $\chi_{3}(u)=c_{3}+O(u), c_{3} \neq c_{1,2}$, or $\chi_{3}(u) \sim c u^{-j}, j>0$. In the latter case the branch $z=\chi_{3}(u)$ meets the line $u=0$ at the point $z=\infty$.

Now we have two possibilities. In the first situation, called the dicritical case, the line $u=0$ ceases to be invariant and all local solutions are analytic, $z=\chi(u)=c+O(u)$. Therefore all solutions $y=\varphi(x)$ of the initial system are regular near $x=0$.

In the non-dicritical case the new Riccati system should have at least 3 singular points on $u=0$ : either 3 finite (i.e. $z=c_{1,2,3}$ ) or 2 finite and one at $z=\infty$. This cannot take place for a Riccati foliation.

REMARK 1. The above proofs run along the lines of [Zo1]. The difference relies on adding the proof of regularity of solutions in the case (d).

REMARK 2. The first integral, of the form (2.4), (2.6) or (2.8), is a multivalued function with ramifications along the lines $x=x_{j}$. Analytic prolongation of its branches along loops in $\mathbb{C}^{2} \backslash \bigcup\left\{x=x_{j}\right\}$ defines its monodromy group $\operatorname{Mon}(H)$. Mon $(H)$ is directly related to the monodromy group of the Riccati equation Mon.

In the case of only one rational solution the monodromy group is infinite and consists of maps $h \rightarrow \lambda_{j} h+\mu_{j}$ (with common fixed point $h=\infty$ corresponding to the solution $y=s(x))$. $\operatorname{Mon}(H)$ is solvable. It is abelian, and consists of translations, when $g \equiv 0$ and $a_{i} \in \mathbb{Z}$ in the generalized Schwarz-Christoffel integral $\int e^{g(u)} \prod\left(u-x_{i}\right)^{a_{i}-m_{i}} P(u)$ in (2.4) (but it is not necessary).

In the case of only two rational solutions the monodromy maps take the form $h \rightarrow \lambda_{j} h$. $\operatorname{Mon}(H)$ can be infinite and finite as well; (but in the latter case $a_{j} \in \mathbb{Q}$ and $g(x) \not \equiv 0$ in $(2.6))$.

In the case with only hypergeometric solution the monodromy maps are of the form $h \rightarrow \lambda_{j} h, h \rightarrow \nu_{j} / h$.

In the case with all solutions algebraic there is no canonical form for the first integral, but the monodromy group of the equation Mon is well defined and finite. Mon can be cyclic (with first integral (2.6) for $g \equiv 0$ and $b_{i} \in \mathbb{Q}$ ), or dihedral (with the first integral $(2.8)$ ), or isomorphic to the group of isometries of the tetrahedron, or the octahedron, or the dodecahedron. 
The vertices of these regular bodies projected onto the sphere $S^{2} \simeq \mathbb{C} P^{1} \times\left\{x_{0}\right\}$ correspond to the branches of the algebraic solution $y=\varphi_{\min }(x)$ with minimal number of branches, equal $\min _{K} \operatorname{deg}_{y} K=4,6$, 12, respectively. We have also $\max \left(\operatorname{deg}_{y} K\right.$, $K$ irreducible) $=12,24,60$ respectively.

3. The polynomial Riccati equations. Denote by $\mathcal{A}_{n} \simeq \mathbb{C}^{n-1}$ the space of Riccati equations of the form

$$
\frac{d y}{d x}=y^{2}-P(x), \quad P(x)=x^{n}+a_{2} x^{n-2}+\ldots+a_{n} .
$$

(By means of changes $x \rightarrow a x+b, y \rightarrow c y$ one can reduce a general Riccati equation $\frac{d y}{d x}=y^{2}-P(x)$ to the form (3.1).)

THEOREM 1. Any possible invariant algebraic curve of (3.1) is of the form

$$
y=T(x)+\sum_{i=1}^{k} \frac{1}{x_{i}-x},
$$

where $T$ is a polynomial of the form

$$
T(x)= \pm x^{m}+b_{2} x^{m-2}+\ldots+b_{m}
$$

and $x_{1}, \ldots, x_{k}$ are different points satisfying the following conditions

$$
T\left(x_{i}\right)+\sum_{j \neq i} \frac{1}{x_{j}-x_{i}}=0, \quad i=1, \ldots, k .
$$

In this case we have

$$
P(x)=T^{2}(x)-T^{\prime}(x)-2 \sum_{i=1}^{k} \frac{T(x)-T\left(x_{i}\right)}{x-x_{i}} ;
$$

(in particular, it must be $n=2 m$ ).

There can be at most one algebraic solution to (3.1). Then there is a first integral of the Darboux-Schwarz-Christoffel type (2.4).

THEOREM 2. The systems from $\mathcal{A}_{n}, n=2 m$ even, with an invariant algebraic curve form a union of countable number of algebraic varietes of dimension $m-1$ each. This means that a generic equation from $\mathcal{A}_{n}$ does not have invariant algebraic curves.

REMARK 3. In [Vi] instead of the representation (3.2) the representation $y=T(x)+$ $F^{\prime} / F$ (obtained previously by Kovacic and van der Put) is used; here $F$ is a monic polynomial with non-zero discriminant. We prefer our formula, because it is more suitable in the following analysis. The last statement in Theorem 1 is absent in [Vi].

Vidunas proves also a result about irreducibility of some algebraic schemes (consisting of the triples $(T, F, P)$ with fixed degrees) under some restrictions. Because at the very beginning we make a normalization (see (3.1)), the corresponding varietes are reducible in our setting.

\section{Proofs}

4.1. Proof of Theorem 1. Let $y=\phi(x), x \in\left(\mathbb{C}, x_{0}\right)$ be a local solution of the equation (3.1). We claim that 
Lemma 1. $\phi(x)$ can be extended to a meromorphic function on $\mathbb{C}$ with simple poles $x_{i}$ such that

$$
\phi(x)=\frac{1}{x_{i}-x}+O(1)
$$

as $x \rightarrow x_{i}$.

Proof. The right-hand side of (4.1) is analytic. Therefore an obstacle to prolongation of the solution $y=\phi(x)$ may be its escaping to infinity. But in the chart $z=1 / y$ near $y=\infty$ we have again the analytic equation

$$
\frac{d z}{d x}=-1+P(x) z^{2} .
$$

Its local solutions through $x=x_{i}, z=0$ are of the form

$$
z=-\left(x-x_{i}\right)+O\left(\left(x-x_{i}\right)^{2}\right)
$$

and are single-valued.

From this, Lemma 1 follows easily.

If a solution $y=\phi(x)$ corresponds to an algebraic curve, then the function $\phi(x)$ must be rational; (this was known already to Liouville [Li]). Let $x_{1}, \ldots, x_{k}$ be the poles of $\phi$. By (4.1) the function

$$
T(x)=\phi(x)-\sum_{i=1}^{k} \frac{1}{x_{i}-x}
$$

is holomorphic on $\mathbb{C}$ with regular growth at infinity. Therefore $T(x)$ is a polynomial and we have the formula (3.2).

We substitute (3.2) to the equation (3.1) and obtain

$$
T^{\prime}=T^{2}-P+2 T \sum \frac{1}{x_{i}-x}+2 \sum_{i<j} \frac{1}{\left(x_{i}-x\right)\left(x_{j}-x\right)} .
$$

We represent the sum $\sum_{i<j}$ as

$$
\sum_{i<j} \frac{1}{x_{j}-x_{i}}\left[\frac{1}{x_{i}-x}-\frac{1}{x_{j}-x}\right]=\sum_{i}\left(\sum_{j \neq i} \frac{1}{x_{j}-x_{i}}\right) \cdot \frac{1}{x_{i}-x}
$$

and (4.2) reads

$$
P-T^{2}+T^{\prime}+2 \sum \frac{T(x)-T\left(x_{i}\right)}{x-x_{i}} \equiv 2 \sum \frac{1}{x_{i}-x} \cdot\left[T\left(x_{i}\right)+\sum_{j \neq i} \frac{1}{x_{j}-x_{i}}\right] .
$$

Since the left-hand side of (4.3) is holomorphic, the right-hand side must vanish and we get condition (3.4) and formula (3.5).

Assuming that $T=b_{0} x^{m}+b_{1} x^{m-1}+\ldots+b_{m}$ and comparing the coefficients of the first two terms in (3.5), we get

$$
b_{0}^{2}=1, \quad 2 b_{0} b_{1}=0
$$

i.e. $T$ is of the form (3.3).

Assume that the equation (3.1) has a solution $y=\phi(x)$ of the form (3.2). We make the change $y_{1}=y-\phi(x)$ and obtain the Bernoulli equation $\frac{d y_{1}}{d x}=2 \phi(x) y_{1}+y_{1}^{2}$. We have 
then the first integral (see $(2.2)$ )

$$
H=\frac{1}{y-\phi(x)} e^{2 \int \phi}+\int e^{2 \int \phi},
$$

where

$$
e^{2 \int \phi}=\frac{e^{2 \int T}}{\prod\left(x-x_{i}\right)^{2}}=\frac{e^{\mathcal{T}(x)}}{\prod\left(x-x_{i}\right)^{2}},
$$

$\mathcal{T}^{\prime}(x)=2 T(x)$. We obtain the first integral in the Darboux-Schwarz-Christoffel form

$$
H=\frac{1}{y-\phi(x)} \cdot \frac{e^{\mathcal{T}(x)}}{\prod\left(x-x_{i}\right)^{2}}+\int^{x} \frac{e^{\mathcal{T}(u)}}{\prod\left(u-x_{i}\right)^{2}} d u .
$$

If there were another rational solution $y=\psi(x)$, then we would have the Darboux integral $H=\frac{y-\psi(x)}{y-\phi(x)} e^{\mathcal{T}(x)} \times$ (rational function in $x$ ) (see the formula (2.6) and its proof). We see that there can be at most two rational solutions (the graphs of other solutions lie in non-algebraic curves $H=h, h \neq 0, \infty)$. Let $\psi(x)=T_{1}(x)+\sum_{j=1}^{l}\left(y_{j}-x\right)^{-1}$. Changing the roles of $\phi$ and $\psi$ in the above analysis, we find that $e^{\mathcal{T}_{1}(x)}=$ const $\cdot e^{-\mathcal{T}(x)}$, i.e. $T_{1}(x) \equiv-T(x)$. The formula (3.4) (for the representation of $P$ ) gives

$$
P=T^{2}-T^{\prime}-2 \sum_{i=1}^{k} \frac{T(x)-T\left(x_{i}\right)}{x-x_{i}}=T^{2}+T^{\prime}+2 \sum_{j=1}^{l} \frac{T(x)-T\left(y_{j}\right)}{x-y_{j}} .
$$

Compare now the coefficients of $x^{m-1}$ :

$$
a_{m+1}=c_{m-1}-(m+2 k)=c_{m-1}+(m+2 l),
$$

where $c_{m-1}$ is the corresponding coefficient associated with $T^{2}(x)$. We see that this is impossible for $k, l \geq 0$.

(Note that the formula (4.5) applied for $n=2 m=2$ gives a necessary condition for the existence of an algebraic solution for the equation $y^{\prime}=y^{2}-x^{2}-a_{0}$ : $a_{0}$ must be an odd integer. Below it is proved that this condition is also sufficient. The same was obtained in [MaMoNo].)

The proof of Theorem 1 is complete.

4.2. Proof of Theorem 2. We shall parametrize the set of equations from $\mathcal{A}_{2 m}$ which possess rational solutions by means of the polynomials $T(x)= \pm x^{m}+b_{2} x^{m-2}+\ldots b_{m}$ and by the couples $\left(x_{1}, \ldots, x_{k}\right), k=0,1,2, \ldots$ of different points satisfying (3.4). The formula (3.5) provides the corresponding parametrizations.

The equation (3.4) defines an algebraic subvariety in $\mathbb{C}^{m-1} \times \mathbb{C}^{k}$ which is a union of irreducible components $\mathcal{B}_{j}$. We have then algebraic maps $F_{j}: \mathcal{B}_{j} \rightarrow \mathcal{A}_{2 m}$ with images $\mathcal{C}_{j}=F_{j}\left(\mathcal{B}_{j}\right)$. The union of these varietes $\mathcal{C}_{j}$ is just the set of systems with an invariant algebraic curve.

Therefore it remains to show that all the varietes have the same dimension $m-1$. The next lemma completes the proof of Theorem 2 .

LemMa 2. For any $\mathcal{B}_{j}$ we have:

(a) $\operatorname{dim} \mathcal{B}_{j}=m-1$.

(b) $\operatorname{dim} \mathcal{C}_{j}=m-1$. 
Proof. The property (a) means that for any polynomial $T(x)$ and any $k$ the system (3.4) has only isolated solutions $\left(x_{1}, \ldots, x_{k}\right)$. Because the first $m+1$ coefficients $1,0, a_{2}, \ldots, a_{m}$ of $P(x)=x^{2 m}+\ldots$ are uniquely expressed by the coefficients $\pm 1,0, b_{2}, \ldots$ $\ldots, b_{m}$ of $T= \pm x^{m}+\ldots$, the map $F_{j}$ is in fact an embedding.

So, it is enough to show that the system (3.4) has only isolated solutions (in finite number). We shall prove even more.

Fix the polynomial $T$. Define the following map $G: \mathbb{C}^{k} \backslash \Delta \rightarrow \mathbb{C}^{k}$ :

$$
\left(x_{1}, \ldots, x_{k}\right) \stackrel{G}{\rightarrow}\left(T\left(x_{1}\right)+\sum_{j \neq 1} \frac{1}{x_{j}-x_{1}}, \ldots, T\left(x_{k}\right)+\sum_{j \neq k} \frac{1}{x_{j}-x_{k}}\right)
$$

here $\Delta=\left\{x: x_{i}=x_{j}\right.$ for some $\left.i \neq j\right\}$ is the union of diagonals $\left\{x_{i}=x_{j}\right\}$. The map $G$ turns out to be proper (recall that the properness of $G$ means that the preimage of any compact subset is a compact set).

The properness of $G$ will follow if we show that:

$$
x \rightarrow\left(\text { boundary of } \mathbb{C}^{k} \backslash \Delta \text { in } \mathbb{C} P^{k}\right) \Rightarrow G(x) \rightarrow\left(\text { boundary of } \mathbb{C}^{k} \text { in } \mathbb{C} P^{k}\right) .
$$

In other words, if $|x| \rightarrow \infty$ or $x \rightarrow \Delta$, then $|G(x)| \rightarrow \infty$.

From (4.6) it would follow that the number of preimages of a point in $\mathbb{C}^{k}$, counted with multiplicities, is constant and the map $G$ is a ramified covering. Therefore $0<$ $\# G^{-1}(0)<\infty$.

Let us prove (4.6). Assume firstly that $|x| \rightarrow \infty$, i.e. $x=\left(x_{1}: \ldots: x_{k}: 1\right) \rightarrow y=\left(y_{1}\right.$ : $\left.\ldots: y_{k}: 0\right) \in H_{\infty}=\mathbb{C} P^{k} \backslash \mathbb{C}^{k}$, but $y_{i} \neq y_{j}$ for $i \neq j$. Then all the summands $\left(x_{j}-x_{i}\right)^{-1} \rightarrow$ 0 , some component $x_{i_{0}} \rightarrow \infty$ and hence $G_{i_{0}}(x)=T\left(x_{i_{0}}\right)+\sum_{j \neq i_{0}}\left(x_{j}-x_{i_{0}}\right)^{-1} \rightarrow \infty$.

Assume that $x \rightarrow y \in \bar{\Delta} \cap H_{\infty}(\bar{\Delta}$ is the closure of $\Delta)$. Suppose that $y_{1}=\ldots=y_{l} \neq 0$ and $y_{i} \neq y_{j}$ if $i \neq j,\{i, j\} \notin\{1, \ldots, l\}$; other cases, when there are several groups of coinciding points are treated analogously. If the distances $\left|x_{i}-x_{j}\right|$ are bounded away from 0 (of order $\sim O(1)$ ), then (as before) we get $G_{i}(x) \rightarrow \infty$ for $i=1, \ldots, m$. If $\max \left\{\left|x_{i}-x_{j}\right|, i \neq j, i, j=1, \ldots, l\right\} \rightarrow 0$, then $x_{1} \rightarrow \infty$ and $x_{i}=x_{1}+o(1), i=2, \ldots, l$ with $x_{1} \rightarrow \infty$. Hence

$$
G_{1}+\ldots+G_{l}=l T\left(x_{1}\right)[1+o(1)]=l x_{1}^{m}+\ldots \rightarrow \infty
$$

(we have used here the identity $\sum\left(x_{i}-x_{j}\right)^{-1}=0$ for the sum over $i, j=1, \ldots, l, i \neq j$ ).

Assume that $x \rightarrow x^{0}=\left(x_{1}^{0}, \ldots, x_{k}^{0}\right) \in \Delta$. Suppose that $x^{0}$ is such that $x_{1}^{0}=\ldots=x_{l}^{0}$ and $x_{i}^{0} \neq x_{j}^{0}$ if $i \neq j,\{i, j\} \notin\{1, \ldots, l\}$; other cases are only technically slightly more complicated. The $l$ points $x_{1}, \ldots, x_{l}$ may focus themselves near the point $x_{1}^{0}$ with different speeds (they would form clusters). We have to look at these points through a magnifying glass, like in the Fulton-MacPherson [FuPh] compactification of the configuration space. In fact, we show properness near any point in the Fulton-MacPherson compactification.

Suppose that all the points $x_{1}, \ldots, x_{l}$ focus themselves near $x_{1}^{0}$ with the same speed, i.e. $x_{j} \rightarrow x_{1}^{0}$ and

$$
x_{2}-x_{1}=\epsilon u_{2}+O\left(\epsilon^{2}\right), x_{3}-x_{1}=\epsilon u_{3}+O\left(\epsilon^{2}\right), \ldots, x_{l}-x_{1}=\epsilon u_{l}+O\left(\epsilon^{2}\right),
$$

$\epsilon \rightarrow 0$, where $\left(u_{2}, \ldots, u_{l}\right)$ belongs to a compact subset $C$ of $\mathbb{C}^{l-1} \backslash \Delta \backslash\left\{u_{2} \cdot \ldots \cdot u_{l}=0\right\}$. 
Then we have $G_{i}=\frac{1}{\epsilon} H_{i}(u)+O(1)$,

$$
H_{1}=\sum_{j=2}^{l} \frac{1}{u_{j}}, H_{i}=-\frac{1}{u_{i}}+\sum_{j \neq i} \frac{1}{u_{j}-u_{i}}, i=2, \ldots, l .
$$

We claim that: at least one of the expressions $H_{i}(u)$ is non-zero.

To show this we notice that

$$
H_{i}=-\frac{1}{2} \frac{\partial}{\partial u_{i}} \log D(u), i=2, \ldots, l,
$$

where $D(u)=u_{2}^{2} \ldots u_{l}^{2} \prod_{i<j}\left(u_{i}-u_{j}\right)^{2}$ is the discriminant of the polynomial $(x-1) \prod(x-$ $\left.1-u_{i}\right)$. The above claim means that: the function $u \rightarrow D(u)$ does not have critical points outside the discriminant locus $\{D(u)=0\}$.

The latter is a consequence of homogeneity of $D$ and the Euler formula $\sum u_{i} \frac{\partial D}{\partial u_{i}}=$ $l(l-1) D$.

If $x_{1}, \ldots, x_{l}$ focus themselves near $x_{1}^{0}$ with different speeds, then we choose a collection $x_{i_{1}}, \ldots, x_{i_{r}}$ with the greatest speed and perform the same analysis.

5. Random matrices. There is a remarkable similarity between the equation (3.4) and a certain formula in the theory of random matrices (see the book of P. Deift [De], for example).

Consider the space of $k \times k$ Hermitian matrices $\mathcal{M}_{k}=\left\{M=\left(m_{i j}\right)_{i, j=1, \ldots, k}: m_{i i} \in\right.$ $\left.\mathbb{R}, m_{i j}=m_{i j}^{R}+\sqrt{-1} m_{i j}^{I}=\overline{m_{j i}}\right\} \simeq \mathbb{R}^{k^{2}}$. Introduce the following probability measure on $\mathcal{M}_{k}$ :

$$
\frac{1}{C} e^{-\operatorname{Tr} \mathcal{T}(M)} \prod_{i} d m_{i i} \prod_{i<j} d m_{i j}^{R} \prod_{i<j} d m_{i j}^{I},
$$

where $C$ is a normalizing constant and $\mathcal{T}(\lambda)$ is a potential. One can represent each Hermitian matrix $M$ in the form $U \cdot \operatorname{diag}\left(\lambda_{1}, \ldots, \lambda_{k}\right) \cdot U^{-1}$, where $U$ is a unitary matrix. Integration over such $U$ 's gives the following measure on $\mathbb{R}^{k}$ (the space of systems of eigenvalues of Hermitian matrices):

$$
\frac{1}{Z} e^{-\sum \mathcal{T}\left(\lambda_{i}\right)} \prod_{i<j}\left(\lambda_{i}-\lambda_{j}\right)^{2} d \lambda_{1} \ldots d \lambda_{k}
$$

where $Z$ is a normalizing factor. Here $\lambda_{i}$ are real and one makes some assumption about the potential: $\mathcal{T}(\lambda)=a \lambda^{2 l}+\ldots, a>0$ (in order that the density be integrable at infinity). The probability distribution (5.2) is the starting point of the theory of random matrices (which has applications in 2-dimensional quantum gravity, in combinatorics, in statistical physics, in the theory of Riemann's zeta function and in many other areas). In the case of quadratic potential one deals with the so-called Unitary Gaussian Ensemble.

The most probable system of eigenvalues $\left(\lambda_{1}, \ldots, \lambda_{k}\right)=\left(\lambda_{1}^{*}, \ldots, \lambda_{k}^{*}\right)$ corresponds to the maximum of the density, i.e.

$$
\min \left[\sum_{i} \mathcal{T}\left(\lambda_{i}\right)-\sum_{i<j} \log \left(\lambda_{i}-\lambda_{j}\right)^{2}\right] .
$$

Such sets $\left\{\lambda_{1}^{*}, \ldots, \lambda_{k}^{*}\right\}$ are called the weighted $k$-Fekete sets (see [De]); there can be several 
such sets. The necessary conditions for $\lambda_{1}^{*}, \ldots, \lambda_{k}^{*}$ are $\left(\right.$ with $\left.\mathcal{T}^{\prime}(x)=2 T(x)\right)$

$$
T\left(\lambda_{i}^{*}\right)-\sum_{j \neq i} \frac{1}{\lambda_{i}^{*}-\lambda_{j}^{*}}=0, \quad i=1, \ldots, k .
$$

These are exactly the equations $(3.4)$.

For quadratic $\mathcal{T}(\lambda)$, i.e. linear $T(\lambda)=\lambda$, the most probable system $\left(\lambda_{1}^{*}, \ldots, \lambda_{k}^{*}\right)$ is such that the polynomial

$$
u(x)=\left(x-\lambda_{1}^{*}\right) \ldots\left(x-\lambda_{k}^{*}\right)
$$

is the monic Hermite polynomial, $u(x)=2^{-k} H_{k}(x)$. (Recall that the Hermite polynomials

$$
H_{k}(x)=k ! \sum_{j=0}^{[k / 2]} \frac{(-1)^{j}(2 x)^{k-2 j}}{j !(k-2 j) !}
$$

are orthogonal polynomials with respect to the measure $e^{-x^{2}} d x$ on $\mathbb{R}$, see [BaEr].)

In $[\mathrm{MaMoNo}]$ the Hermite equation

$$
u^{\prime \prime}-2 x u^{\prime}+2 k u=0
$$

has occurred (but it was not recognized as the Hermite equation). The other equation from [MaMoNo], i.e. $u^{\prime \prime}+2 x u^{\prime}-2 k u=0$ (corresponding to the non-physical potential $\left.\mathcal{T}=-x^{2}\right)$ has the solution const $\cdot H_{k}(\sqrt{-1} x)$.

In the general case, instead of (5.4), one gets the equation

$$
u^{\prime \prime}-2 T(x) u^{\prime}+2\left(\sum \frac{T(x)-T\left(x_{i}\right)}{x-x_{i}}\right) u=0
$$

(it is the same as the equation (1.3) in [Vi]). If $\operatorname{deg} T \geq 2$, then this equation becomes nonlinear for $u=\left(x-x_{1}\right) \ldots\left(x-x_{k}\right)$. Moreover, the system (5.4) may have several solutions (not as in the case of orthogonal polynomials, which are unique in the real domain).

In the book of P. Deift the probability measure (5.2) and the extremum problem (5.3)) are investigated from the asymptotic point of view for $k \rightarrow \infty$. The following result about weighted $k$-Fekete sets was proved in [De, Theorem 6.62].

Assume that together with $k \rightarrow \infty$ we scale the potential, $\mathcal{T}(\lambda)=(k-1) \mathcal{T}_{0}(\lambda)$. Then the discrete measures $\frac{1}{k} \sum \delta_{\lambda_{j}^{*}}$ supported on the weighted $k$-Fekete sets $\left\{\lambda_{1}^{*}, \ldots, \lambda_{k}^{*}\right\}$ converge weakly to the so-called equilibrium measure $\mu=\mu^{\mathcal{T}_{0}}$ uniquely defined by the condition that the quantity

$$
\int \mathcal{T}_{0}(x) d \mu(x)-2 \iint \log |x-y| d \mu(x) d \mu(y)
$$

is minimal.

In [De] it is proved that also the discrete measures $\frac{1}{k} \sum \delta_{z_{j}}$ supported in the roots of the $k$-th monic polynomials $u_{k}(x)$ orthogonal with respect to the measure $e^{-k \mathcal{T}_{0}(x)} d x$ converge to the measure $\mu^{\mathcal{T}_{0}}$. Moreover, the measure $\mu^{\mathcal{T}_{0}}$ turns out to be absolutely continuous with respect to the Lebesgue measure and to have compact support. For example, when $\mathcal{T}_{0}(\lambda)=\lambda^{2}$, the measure $\mu^{\mathcal{T}_{0}}$ is supported in $[-\sqrt{2}, \sqrt{2}]$, has density 
$\frac{1}{\pi} \sqrt{2-x^{2}}$ and the corresponding limit theorem about eigenvalues of random Hermitian matrices is called Wigner's semicircle law.

Using these results we can say something about asymptotic properties of solutions of polynomial Riccati equations with a large number of poles.

Theorem 3. Assume that $m=n / 2$ is odd. Let us put $T(x)=(k-1) T_{0}(x)$ for a fixed polynomial $T_{0}=x^{m}+\ldots$ with real coefficients and the integer $k \rightarrow \infty$.

Then the real poles $x_{1}^{*}, \ldots, x_{k}^{*}$ (of the solution (3.2)), defined by the system (3.5) and by the condition that the quantity $\left[\sum \mathcal{T}\left(x_{i}^{*}\right)-\sum_{i<j} \log \left(x_{i}^{*}-x_{j}^{*}\right)^{2}\right]$ is minimal, become uniformly distributed with respect to the measure $\mu^{\mathcal{T}_{0}}$. In particular, they become concentrated in a compact subset of $\mathbb{R}$.

I think that this direction of studying Riccati systems is interesting and deserves further investigation. For example, it would be desirable to know whether an analogue of Theorem 3 holds in the case of general polynomial $T_{0}$ (e.g. with complex coefficients).

Acknowledgments. I thank J. A. Weil for his comments on the first version of my paper and the referee for suggestions which have improved the presentation of the work. I thank also Janek Wehr for the book of Deift.

\section{References}

[BaEr] H. Bateman and A. Erdélyi, Higher Transcendental Functions, v. 2, McGraw-Hill, New York, 1953.

[De] P. Deift, Orthogonal Polynomials and Random Matrices: a Riemann-Hilbert Approach, Courant Lect. Notes in Math. 3, Courant Inst. Math. Sci., New York, 1999.

[FuPh] W. Fulton and R. MacPherson, Compactification of configuration spaces, Ann. Math. 139 (1994), 183-225.

[Ka] I. Kaplansky, An Introduction to Differential Algebra, Hermann, Paris, 1957.

[Ko] J. Kovacic, An algorithm for solving second order linear homogeneous differential equations, J. Symb. Comput. 2 (1986), 3-43.

[MaMoNo] A. Maciejewski, J. Moulin-Ollagnier and A. Nowicki, Simple quadratic derivations in two variables, Comm. Algebra 29 (2001), 5095-5113.

[Li] J. Liouville, Mémoire sur l'intégration d'une classe d'équations différentielles du second ordre en quantités explicites, J. Math. Pures Appl. 4 (1841), 425-456.

[Mo] J. J. Morales-Ruiz, Differential Galois Theory and Non-Integrability of Hamiltonian Systems, Progr. Math. 179, Birkhäuser, Boston, 1999.

[Vi] R. Vidunas, Differential equations of order two with one singular point, J. Symb. Comput. 28 (1999), 495-520.

[Zo1] H. Żołądek, The classification of reversible cubic systems with center, Topol. Methods Nonlin. Anal. 4 (1994), 79-136.

[Zo2] H. Żołądek, Algebraic invariant curves for the Liénard equation, Trans. Amer. Math. Soc. 350 (1998), 1681-1701.

[Zo3] H. Żołądek, The method of holomorphic foliations in planar periodic systems. The case of Riccati equations, J. Diff. Equat. 165 (2000), 143-173. 